This item was submitted to Loughborough's Research Repository by the author.

Items in Figshare are protected by copyright, with all rights reserved, unless otherwise indicated.

\title{
Two-dimensional simulation of a low-current dielectric barrier discharge in atmospheric helium
}

PLEASE CITE THE PUBLISHED VERSION

PUBLISHER

(c) American Institute of Physics

VERSION

VoR (Version of Record)

LICENCE

CC BY-NC-ND 4.0

\section{REPOSITORY RECORD}

Zhang, Yuan Tao, De Zhen Wang, and Michael G. Kong. 2019. "Two-dimensional Simulation of a Low-current Dielectric Barrier Discharge in Atmospheric Helium”. figshare. https://hdl.handle.net/2134/5174. 
This item was submitted to Loughborough's Institutional Repository (https://dspace.lboro.ac.uk/) by the author and is made available under the following Creative Commons Licence conditions.

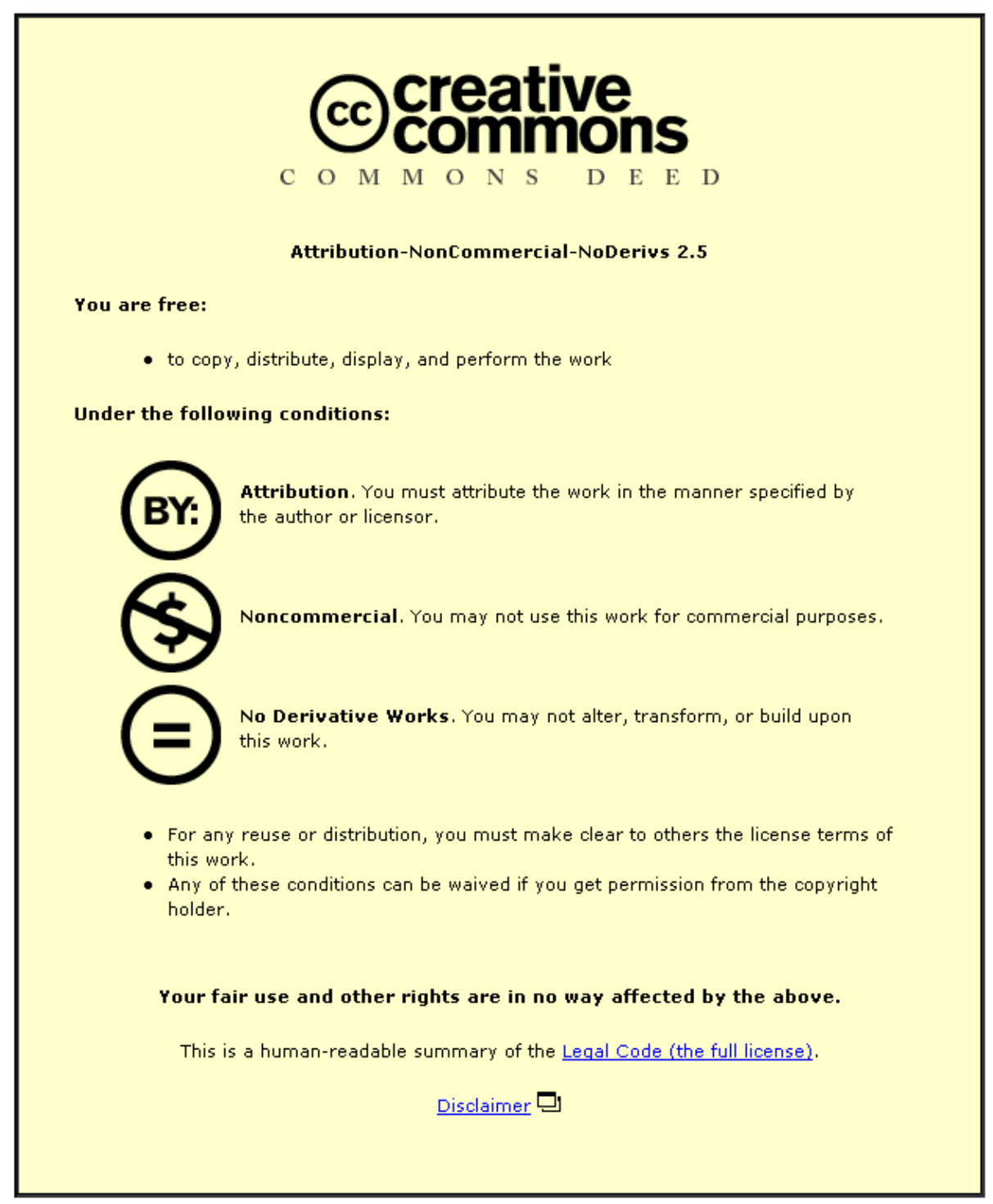

For the full text of this licence, please go to: http://creativecommons.org/licenses/by-nc-nd/2.5/ 


\title{
Two-dimensional simulation of a low-current dielectric barrier discharge in atmospheric helium
}

\author{
Yuan Tao Zhang and De Zhen Wang ${ }^{\text {a) }}$ \\ State Key Laboratory of Materials Modification by Laser, Ion and Electron Beams, Department of Physics, \\ Dalian University of Technology, Dalian, People's Republic of China \\ Michael G. Kong \\ Department of Electronic and Electrical Engineering, Loughborough University, Leicestershire LE11 3TU, \\ United Kingdom
}

(Received 7 February 2005; accepted 3 November 2005; published online 15 December 2005)

\begin{abstract}
A two-dimensional computational study is presented to unravel radial structure of a dielectric barrier discharge in atmospheric helium when the gas voltage exceeds slightly the breakdown voltage and the discharge current is low to retain a repetitive dynamic pattern of one discharge event every half cycle of the applied voltage. Simulation results reveal that during each half cycle of the applied voltage gas breakdown occurs first in a central region around the electrode axis. After it is extinguished, a second breakdown is triggered in the boundary region near the radial edge of the two electrodes as confirmed by the dynamic evolution of the radial profile of the electric field, the current density and the charged particles. These predictions are consistent with relevant experimental observations in literature. It is also shown that an increase in the applied voltage or in the excitation frequency reduces the time delay between the two breakdown events and the difference between their corresponding current densities. This offers a route to improve the uniformity of atmospheric dielectric barrier discharges for their intended applications. (C) 2005 American Institute of Physics. [DOI: 10.1063/1.2140890]
\end{abstract}

\section{INTRODUCTION}

Atmospheric gas discharges controlled by dielectric barrier have recently attracted much attention because of their widespread industrial applications, such as thin film deposition, surface modification, sterilization of biological samples, and etching. Usually these atmospheric dielectric barrier discharges consist of numerous short-lived filaments each on a nanosecond scale. With effective control of their discharge current, their appearance becomes homogeneous filling up the entire space between their two electrodes. Such homogeneous atmospheric discharges have greater spatial uniformity, better temporal stability, and much lower gas temperature than what is typical in filamentary atmospheric discharges. This regime of atmospheric discharges can be realized in different gases but most commonly in helium and nitrogen. ${ }^{1-3}$ They are often known as atmospheric pressure glow discharge (APGD). Basic understanding of APGD has been considerably advanced through extensive experimental and theoretical studies since they were first reported. ${ }^{1}$

Typical dynamic behaviors of APGD controlled by dielectric barriers are characterized by a single current peak every half-cycle of the applied voltage. ${ }^{2,3}$ This occurs when the voltage across the gas gap is slightly above the gas breakdown voltage and the discharge current density is modest. With a larger gap voltage, it is possible to trigger several discharge events every half-cycle of the applied voltage and the peak density of these discharge events is markedly higher than that in the case with one discharge event every

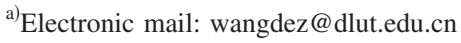

half-cycle. ${ }^{4,5}$ This study considers APGD with modest current densities and with only one discharge event every halfcycle of the applied voltage.

Given the apparent homogeneous appearance of APGD, their numerical simulation has so far been based on a onedimensional (1D) model that implicitly assumes a radial uniformity. ${ }^{2,3,6}$ However radial structure of APGD has been observed experimentally, ${ }^{7}$ in particular their radial dynamics are characterized by a first discharge that is ignited in the central region around the electrode axis and then spreads towards the electrode edge. Subsequently a second discharge occurs at the periphery of the electrodes. ${ }^{7}$ This highlights the considerable radial variation in APGD dynamics and a clear need to account for radial profiles of plasma densities. Experimentally electron density below $10^{11} \mathrm{~cm}^{-3}$ is difficult to measure ${ }^{8}$ while an electron density of $10^{9}-10^{12} \mathrm{~cm}^{-3}$ is common in APGD. ${ }^{2,3,6}$ To unravel 2D distribution of plasma densities and their dynamics, an experimental approach is therefore ineffective and a numerical approach is more appropriate. So far most numerical simulations of helium APGD have been confined to one dimension. ${ }^{2,4-6,9,10}$ In this contribution, we present a two-dimensional (2D) fluid model in a cylindrical symmetric coordinate system to study the radial evolution of APGD when the discharge current density is low $\left(<10 \mathrm{~mA} / \mathrm{cm}^{2}\right)$ and there is one current peak every half cycle of the applied voltage.

\section{MODEL AND ELEMENT PROCESSES}

\section{A. Model description}

We consider APGD in a pure helium gap between two dielectrically coated electrodes. While impurities in the back- 
ground helium gas are known to be important, 2,9 our objective here is to establish the generic dynamic evolution in 2D rather than a quantitative comparison with a particular experiment. So we will leave the effect of impurities to a future study. Our numerical simulation is based on a 2D fluid model. The particles we take into account include electrons, ions $\left(\mathrm{He}^{+}, \mathrm{He}_{2}^{+}\right)$and neutral particles $\left(\mathrm{He}, \mathrm{He}^{*}\right)$. Within the scope of the diffusion-drift approximation, the charged particles can be described by the continuity equations in cylindrical geometry

$$
\begin{aligned}
& \frac{\partial n_{k}}{\partial t}+\frac{1}{r} \frac{\partial\left(r j_{k r}\right)}{\partial r}+\frac{\partial\left(j_{k z}\right)}{\partial z}=S_{k}, \\
& j_{k r}= \pm \mu_{k} E_{r} n_{k}-D_{k} \frac{\partial n_{k}}{\partial r} \\
& j_{k z}= \pm \mu_{k} E_{z} n_{k}-D_{k} \frac{\partial n_{k}}{\partial z}
\end{aligned}
$$

where $n_{k}$ is the density of the corresponding particles and $S_{k}$ is the source term of these particles, including their production and loss. $j_{k}$ is the current density, and $\mu_{k}$ and $D_{k}$ are the mobility and diffusion coefficients, respectively. The minus sign in Eqs. (2) and (3) is taken for electrons, and the plus sign for positive particles. As for the neutral particle, only the diffusion item is effective. The indices $r$ and $z$ correspond to radial and axial components, respectively. The detailed chemical processes will be described in the next subsection.

For the calculation of the electric field, the Poisson equation is solved. In the cylindrical coordinate, it is represented as

$$
\frac{1}{r} \frac{\partial\left(r E_{r}\right)}{\partial r}+\frac{\partial E_{z}}{\partial z}=\frac{\rho}{\varepsilon_{0}},
$$

where $E_{r}$ and $E_{z}$ are the radial and axial component of the electric field. $\rho$ is the space charge density. It is well known that numerical solution of the Poisson equation in a 2D space is a complicated and time-consuming procedure. So in our model we replace the Poisson equation by the current balance equation in the cylindrical geometry

$$
\begin{aligned}
& \frac{\partial E_{r}}{\partial t}+\frac{e}{\varepsilon_{0}} j_{r}=\frac{e}{\varepsilon_{0}} j_{0 r}, \\
& \varepsilon(z) \frac{\partial E_{z}}{\partial t}+\frac{e}{\varepsilon_{0}} j_{z}=\frac{e}{\varepsilon_{0}} j_{0 z},
\end{aligned}
$$

where $j_{r}$ and $j_{z}$ are, respectively, the radial and axial components of the conduction current density. $j_{0 r}$ and $j_{0 z}$ are the radial and axial components of the total current density, respectively. $\varepsilon(z)$ is the permittivity of either the dielectric or the gas gap depending on the position $z$. In our calculation, Eqs. (1)-(3) are discretized and solved with the SchartetterGummel scheme. ${ }^{11}$ It has been established that the current balance equations of (5) and (6) lead to the same electric field distribution as that solved directly from the Poisson equation provide that the initial conditions of the space charges and the electric field satisfy the Poisson equation. ${ }^{12,13}$ This technique was successfully used to study the low-frequency and high-pressure Xe barrier discharges for the design of excimer lamps. ${ }^{14}$ In our numerical scheme, the whole computational region is divided into $300 \times 100$ uniform grids. A typical CPU time of our computer code to reach a stable state of is about $1 \frac{1}{2}$ days according to our scheme on a $3.0 \mathrm{GHz}$ Pentium $4 \mathrm{PC}$.

The axial total current is composed of the displacement current and the conduction current, and can be represented as

$$
j_{0 z}=\frac{\varepsilon_{0}}{e\left(d+\frac{2 d_{b}}{\varepsilon_{b}}\right)} \frac{\partial V_{0}(t)}{\partial t}+\frac{1}{d+\frac{2 d_{b}}{\varepsilon_{b}}} \int_{0}^{d} j_{z} d z,
$$

where $d_{b}$ is the thickness of the dielectric barrier, $\varepsilon_{b}$ is its permittivity, and $d$ is the distance between the two dielectric barriers. $V_{0}(t)$ is the applied voltage. The radial component of the total current density is given by Refs. 12 and 13

$$
j_{0 r}=0 \text {. }
$$

The basic equations in our model are Eqs. (1)-(3) and (5)-(8), in which there are seven unknowns, namely, $n_{k}, j_{k r}$, $j_{k z}, E_{r}, E_{z}, j_{0 r}$, and $j_{0 z}$. The total current densities of $j_{0 r}$ and $j_{0 z}$ satisfies

$$
\nabla \cdot \mathbf{j}_{0}=0
$$

throughout the entire space between the two electrodes. ${ }^{12,13}$ Their solutions are given analytically in Eqs. (7) and (8), and do not require boundary conditions. To numerically compute $n_{k}, j_{k r}, j_{k z}, E_{r}$, and $E_{z}$ the space between the two electrodes is discretized using a $2 \mathrm{D}$ grid. In the resulting computational domain, densities of plasma species are obtained at integer nodes whereas current densities and the electric field are calculated at half-integer nodes, as detailed in literature. ${ }^{11-13}$ The calculation begins with a spatially uniform density for ions and electrons both set at $10^{7} \mathrm{~cm}^{-3}$. The diffusion of surface charges on the dielectrically coated electrodes is ignored in our calculation because the motion of diffusion relates to the surface curvature ${ }^{15}$ and this finer feature will be considered in future. The emission of secondary electrons from the insulator is important for dielectric barrier discharges and should be included. As a simple first step, we consider ion bombardment only and assume the corresponding secondary emission coefficient to be $\gamma=0.01$. Therefore the electron density leaving the electrodes is given by $\gamma j_{k y}$ with $j_{k y}$ being the current densities of incoming helium ions $\left(\mathrm{He}^{+}\right.$and $\left.\mathrm{He}_{2}^{+}\right)$.

\section{B. Elementary process}

For our simulation conditions, i.e., at atmospheric pressure and with low ionization degree, some processes are much faster than the characteristic time of glow discharge evolution. We can therefore simplify the kinetics of excitation and ionization in helium. The ionization and excitation species included in this model are atomic ion $\mathrm{He}^{+}$, molecular ion $\mathrm{He}_{2}^{+}$and the main metastables $\mathrm{He}\left(2^{3} S\right)$ and $\operatorname{He}\left(2^{1} S\right)$. Since at atmospheric pressure the metastable states $\mathrm{He}\left(2^{3} S\right)$ and $\operatorname{He}\left(2^{1} S\right)$ have very similar behavior, we group them together into one effective species, $\mathrm{He}^{*}$. The elementary processes included in the source term are listed in Table I. The 
TABLE I. The elementary processes in the model of helium plasma.

\begin{tabular}{|c|c|c|c|}
\hline Reaction name & Reaction equation & Rate constant & Reference \\
\hline Direct ionization & $e+\mathrm{He} \rightarrow \mathrm{He}^{+}+2 e$ & $\alpha$ & 18 \\
\hline Excitation & $e+\mathrm{He} \rightarrow \mathrm{He}^{*}+e$ & $4.2 \times 10^{-9} T_{e}^{0.31} e^{-19.8 / T_{e}} \mathrm{~cm}^{3} / \mathrm{s}$ & 19 \\
\hline Ionization of metastable & $e+\mathrm{He}^{*} \rightarrow \mathrm{He}^{+}+2 e$ & $1.28 \times 10^{-7} T_{e}^{0.6} e^{-4.78 / T_{e}} \mathrm{~cm}^{3} / \mathrm{s}$ & 19 \\
\hline Ion conversion & $\mathrm{He}++2 \mathrm{He} \rightarrow \mathrm{He}_{2}^{+}+\mathrm{He}$ & $2.0 \times 10^{-31} \mathrm{~cm}^{3} / \mathrm{s}$ & 19 \\
\hline Penning ionization & $\mathrm{He}^{*}+\mathrm{He}^{*} \rightarrow e+\mathrm{He}^{+}+\mathrm{He}$ & $8.7 \times 10^{-10} \mathrm{~cm}^{3} / \mathrm{s}$ & 20 \\
\hline & $\rightarrow e+\mathrm{He}_{2}^{+}$ & $2.03 \times 10^{-9} \mathrm{~cm}^{3} / \mathrm{s}$ & \\
\hline \multirow[t]{3}{*}{ Dissociative recombination } & $\mathrm{He}_{2}^{+}+e \rightarrow 2 \mathrm{He}^{*}+\mathrm{He}$ & $5.9 \times 10^{-9} \mathrm{~cm}^{3} / \mathrm{s}$ & 21 \\
\hline & \multirow[t]{2}{*}{$\mathrm{He}^{+}+e \rightarrow \mathrm{He}$} & & \multirow[t]{2}{*}{17} \\
\hline & & $8.1 \times 10^{-20} \frac{n_{e}}{1+0.079 p}\left(T_{e} / T_{g}\right)^{-4.4} \mathrm{~cm}^{3} / \mathrm{s}$ & \\
\hline Deexcitation & $\mathrm{He}^{*}+\rightarrow \mathrm{He}+e$ & $2.9 \times 10^{-9} \mathrm{~cm}^{3} / \mathrm{s}$ & 20 \\
\hline
\end{tabular}

mobility and diffusion coefficients for electron and ion $\mathrm{He}^{+}$ are obtained from Ref. 16 and Ref. 17, respectively. We obtain the mobility and diffusion coefficients for $\mathrm{He}_{2}^{+}$and the metastable diffusion coefficients from Ref. 17. The direct ionization coefficient is obtained using Ward's formula. ${ }^{18}$

\section{RESULT AND DISCUSSION}

For all numerical examples presented here, the gap between the two electrodes is $3 \mathrm{~mm}$ and the thickness of each dielectric layer is $1 \mathrm{~mm}$ with their permittivity at 7.6. The electrode radius is set to $5 \mathrm{~mm}$ and the driving frequency of the applied sinusoidal voltage is $20 \mathrm{kHz}$. The working gas is helium at 760 Torr. When the amplitude of the applied voltage exceeds only slightly the breakdown voltage, a single current peak is observed during each half cycle. The profile of the space-averaged current density over the whole electrode surface and the identically space-averaged gap voltage is presented in Fig. 1. This is very similar to that obtained experimentally $^{1,2}$ and using a 1D simulation. ${ }^{4,5}$ The main characteristics of a repetitive pattern with one current peak have been discussed in detail by many authors, but the radial distribution of the current density has not been discussed numerically because the reported numerical studies have so far been restricted to one dimension. With our 2D code, the radial distribution of the current density at different radial positions is obtained and presented in Fig. 2. From the radial distribution of the current density, two characters of the radial evolution of APGD are evident. First, the discharge current densities at different $r$ do not reach their peaks simultaneously. In the central region of $r=0-4.2 \mathrm{~mm}$, gas breakdown takes place first and the discharge current density reaches its peak earlier than that in the boundary region $(r$ $=4.2-5 \mathrm{~mm}$ ). In other words, there exists a time delay between the plasma ignition in the central region and in the boundary region. Second, the amplitude of the current density decays radially. The radial distribution in Fig. 2 means that the discharge is stronger in the central region than in the boundary. To show the radial profile of the generated plasma clearly, the whole discharge area can be divided into two regions, namely, the central region $(0-4.2 \mathrm{~mm})$ and the boundary region $(4.2-5 \mathrm{~mm})$. The average current densities of the central region and the boundary region are illustrated in Fig. 3, together with the average total current density. It is evident from Fig. 3 that the overall space-averaged current density is slightly smaller than that in the central region.

In order to understand the radial evolution of APGD better, Fig. 4 is used to show spatial distributions of ion and electron densities at two key moments. When the gas breakdown occurs in the central region at $t=207.25 \mu$ s, Figs. 4(a)

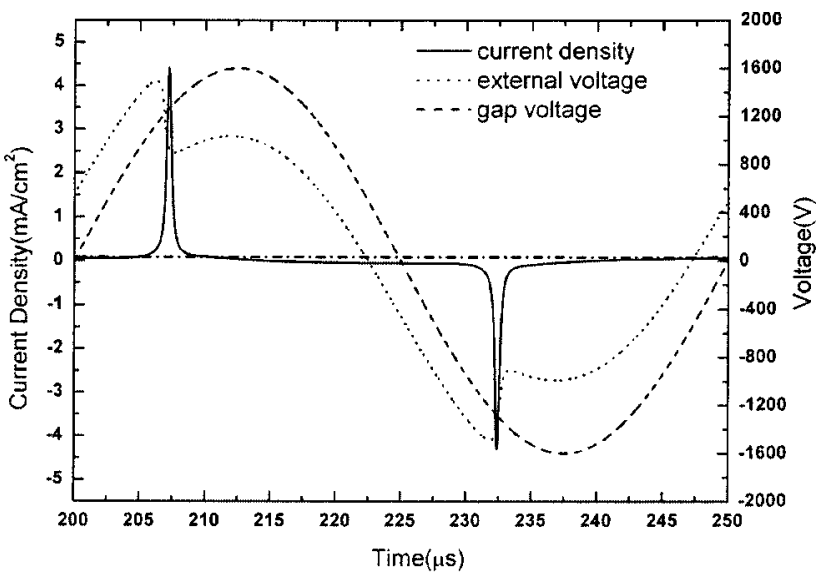

FIG. 1. The profiles of the overall average current density, the applied voltage, and the gas voltage. The amplitude of the applied voltage is $1600 \mathrm{~V}$ and the driving frequency is $20 \mathrm{kHz}$.

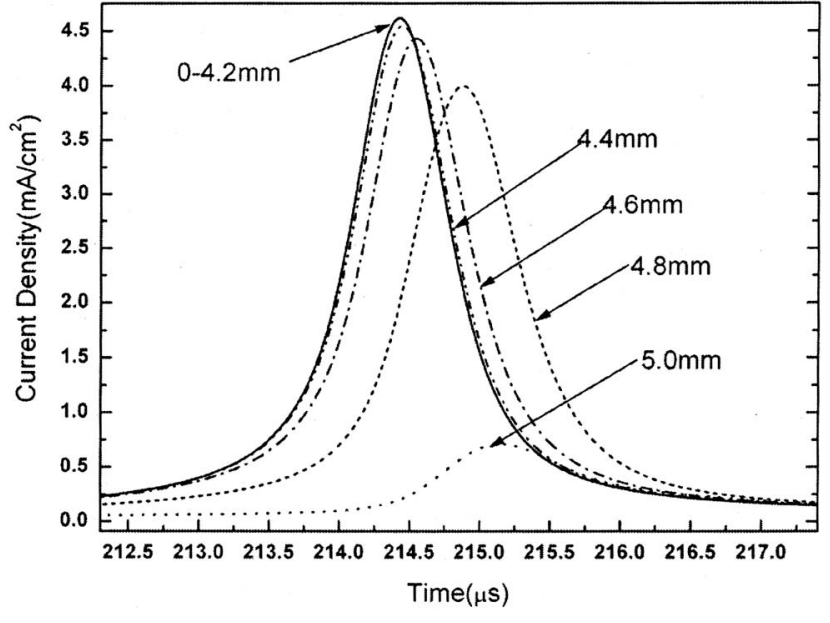

FIG. 2. The current densities with different radial positions. 


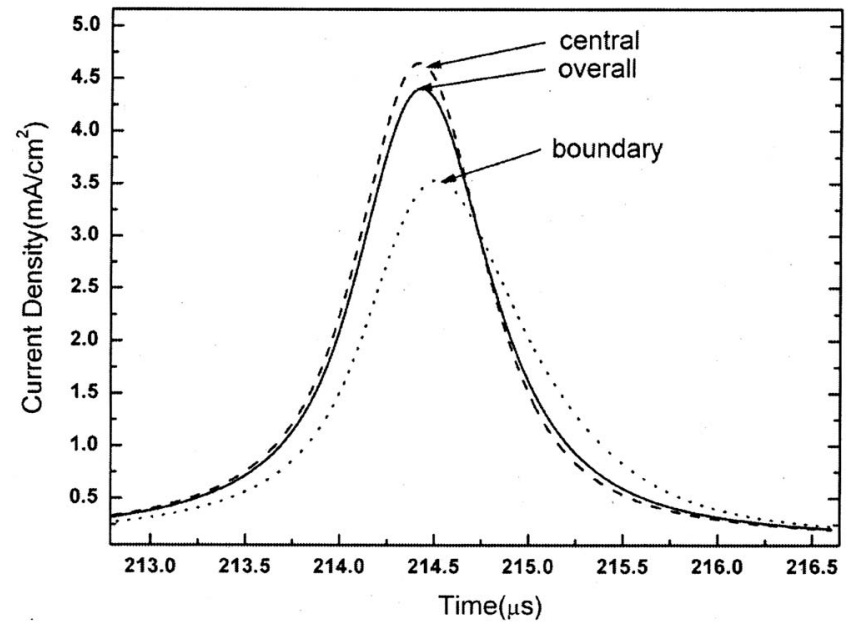

FIG. 3. Temporal dependence of the current density and the current density in the central and boundary regions.

and 4(c) show that both the electron and ion densities are much stronger in the central region. This is a result of the gas breakdown being confined to the central region and so not uniform radially. After the discharge is formed from this centrally confined gas breakdown, it begins to expand towards the electrode boundary. Consequently a new gas breakdown occurs outside the central region, and progressively this new gas breakdown appears to propagate outwards radially from $r=4$ to $5 \mathrm{~mm}$. This radial propagation is associated with a progressive reduction of the electron and ion densities in the central region and a simultaneous increase in particle densities in the boundary region. In Figs. 4(b) and 4(d), the electron and ion densities are seen to be largest near $r=4.7 \mathrm{~mm}$ in the boundary region at $t=207.4 \mu$ s when the second breakdown occurs.

The mechanism responsible for the radial evolution of APGD in Fig. 4 can be understood from the radial electric field distribution in Fig. 5 and the dynamics of the surface charge density and the gap voltage in Fig. 6. At $t$ $=207.25 \mu$ s the electric field in Fig. 5(a) is clearly larger in
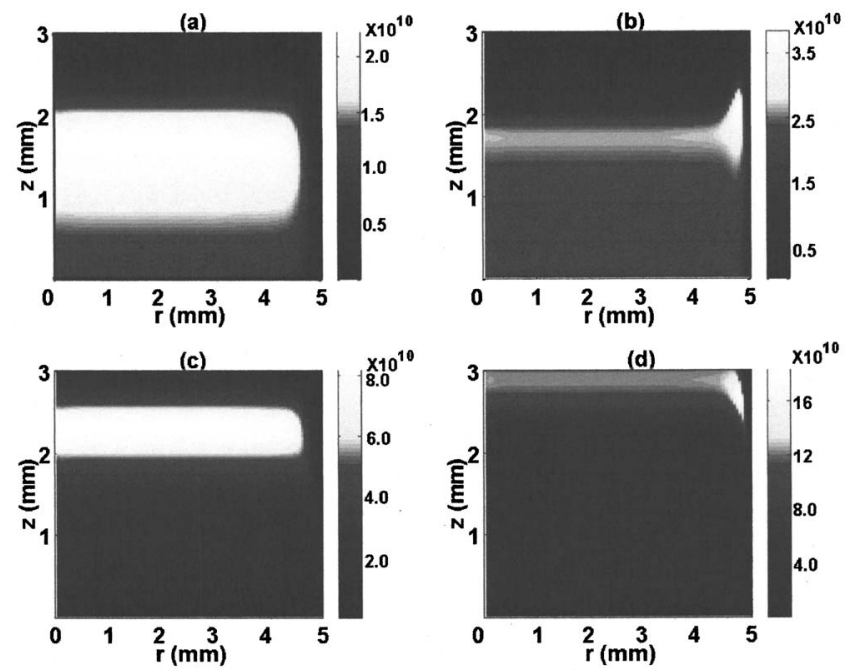

FIG. 4. Ion density profiles at (a) $207.25 \mu$ s and (b) $207.4 \mu$ s. Electron density profiles at (c) $207.25 \mu \mathrm{s}$, and (d) $207.4 \mu \mathrm{s}$. The momentary cathode is located at the top in each picture.
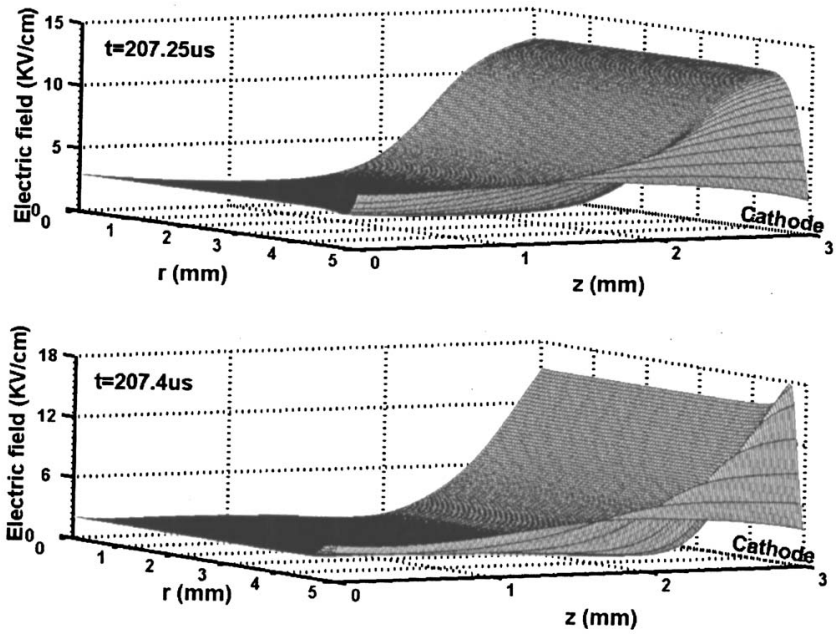

FIG. 5. Total electric field profiles at (a) $207.25 \mu$ s and (b) $207.4 \mu \mathrm{s}$.

the central region and this is directly related to the large current density for $r=0-4.2 \mathrm{~mm}$ in Fig. 2. The small electric field in the boundary region of Fig. 5(a) is also consistent with the very low current density for $r=4.6-5 \mathrm{~mm}$ at the same time. Similarly at $t=207.4 \mu \mathrm{s}$, the electric field in Fig. 5(b) shows a clear maximum of almost $18 \mathrm{kV} / \mathrm{cm}$ at $r$ $=4.6 \mathrm{~mm}$ and this large electric field triggers gas breakdown in the boundary region as identified by the current density peak at $t=207.4 \mu$ s for $r=4.6 \mathrm{~mm}$ in Fig. 2. It is evident that the electric field in the boundary region has a radial profile that decays rapidly away from its peak. As a result the gas breakdown is likely to be triggered within a radially confined region and so the space average of the induced discharge current for the boundary region is much smaller than the current density at the location of the maximum electric field. This contrasts the apparently uniformity in the radial distribution of the electric field in the central region of Fig. 5(a) for $t=207.25 \mu \mathrm{s}$. The different radial profiles of the electric field in the central and boundary regions are responsible for the difference in the current densities in Fig. 3, even though the maximum electric field in Fig. 5(b) is greater than that in Fig. 5(a).

The above interpretation can be further supported by

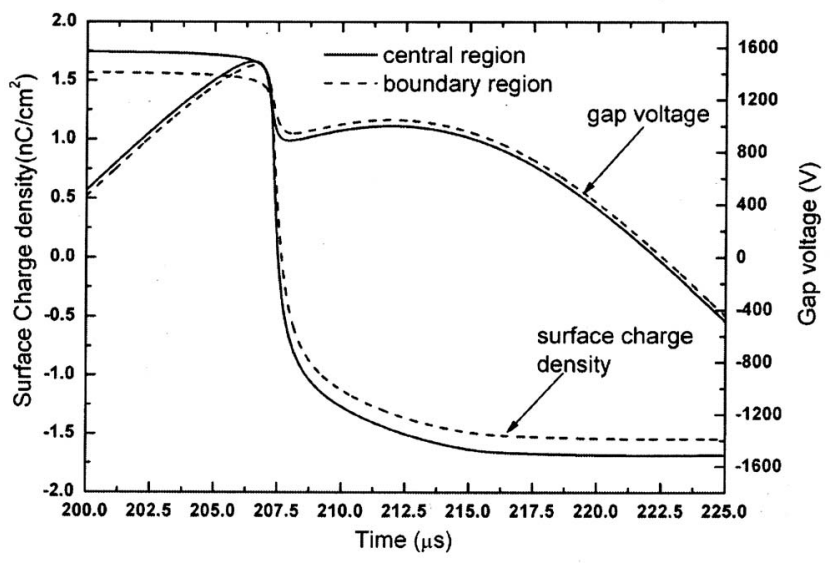

FIG. 6. The profiles of surface charge density and the gap voltage in the central and boundary region. 

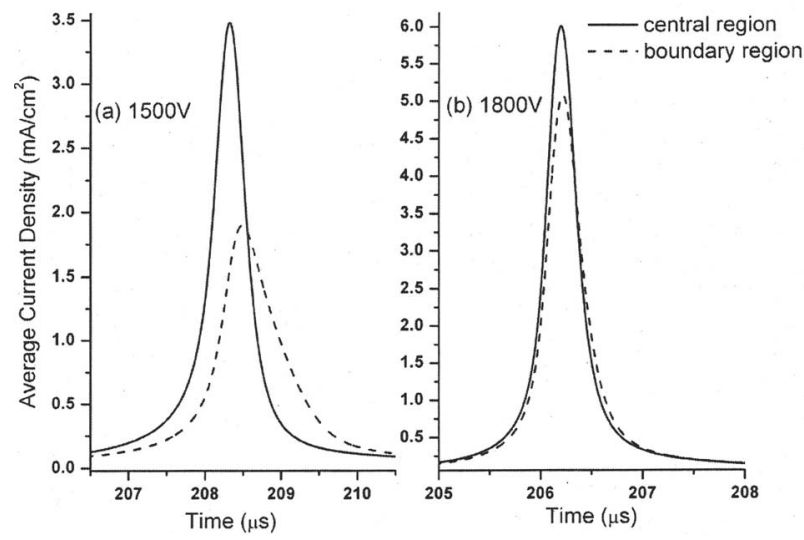

FIG. 7. The profiles of the current densities at the central and boundary regions with the amplitude of the applied voltage at (a) $1500 \mathrm{~V}$ and (b) $1800 \mathrm{~V}$.

Fig. 6 where the surface charge density on the dielectric layer and the space-averaged gap voltage are plotted as a function of time. In Fig. 6, the gap voltage profiles suggest that when a new half-cycle begins, the gap voltage in the central region is bigger than that in the boundary region until the breakdown occurs. After the ignition however, the gap voltage in the central region become smaller than that in the boundary region. This observation indicates that the total electric field near the boundary region may be stronger than that in the central region after the breakdown. So if the applied voltage is strong enough to turn on new avalanche, the second current peak is more likely to be formed in the boundary region. The same dynamic evolution is also indicated from the profile of surface charge density, and this highlights the important role of surface charge in the radial variation of APGD as discussed above.

To understand how plasma operation conditions may influence the radial variation, we introduce a ratio number $U 1$ :

$$
U 1=\frac{A_{c}-A_{b}}{A_{c}},
$$

where $A_{c}$ and $A_{b}$ are the amplitudes of the space-averaged current densities for the central and the boundary regions, respectively. From this definition, a small value of $U 1$ means an insignificant variation in the current density from the central to boundary regions. Similarly the instants of gas breakdown in the central and boundary regions can be compared by introducing a second ratio parameter $U 2$ as follows:

$$
U 2=\frac{t_{c}-t_{b}}{T},
$$

where $t_{c}$ and $t_{b}$ are, respectively, the moments when the current density reaches its maximum in the central and the boundary regions. $T$ is the period of the applied voltage. A small value of $U 2$ suggests a short time delay between the two radially separated discharge events.

Figure 7 shows the time evolution of space-averaged current densities with different applied voltages. All other parameters are kept unchanged. The applied voltage of Figs. 7(a) and 7(b) has the amplitude of $1500 \mathrm{~V}$ and $1800 \mathrm{~V}$, respectively. From Fig. 7 it is immediately clear that as the

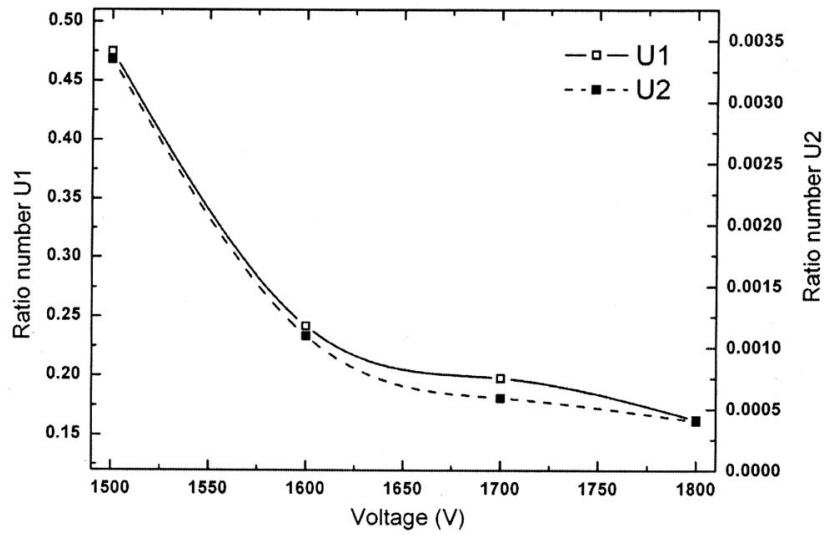

FIG. 8. The profiles of the ratio numbers $U 1$ and $U 2$ as a function of the amplitude of the applied voltage with the driving frequency fixed at $20 \mathrm{kHz}$.

applied voltage increases the difference in the discharge current densities becomes smaller. The time delay also become smaller. The diagrams of $U 1$ and $U 2$ display these results directly as shown in Fig. 8. Relating the observations of Figs. 7 and 8 back to the underlying physics of APGD, it is clear that a large applied voltage compensates a small memory voltage in the boundary region to trigger gas breakdown there earlier. A large applied voltage is likely to force gas breakdown across a wider radial expansion from the electrode axis and ultimately enable simultaneous gas breakdown over the entire radial extent of the electrodes. Another consequence of an increased applied voltage is a wider central region and a proportionally smaller boundary region.

The $U 1$ profile can also be used to understand the effect of the driving frequency and this is shown in Fig. 9 for which the amplitude of the applied voltage is kept at $1600 \mathrm{~V}$. As the frequency increase, $U 1$ reduces rapidly first and then its reduction slows down for frequencies above $30 \mathrm{kHz}$. From a practical standpoint, Figs 8 and 9 suggest that APGD are likely to be more uniform radially with a large applied voltage provided that it is not too large to induce the glow-to-arc transition and the excitation frequency is not too high to induce electron trapping. ${ }^{22}$ The trends shown in Figs. 8 and 9

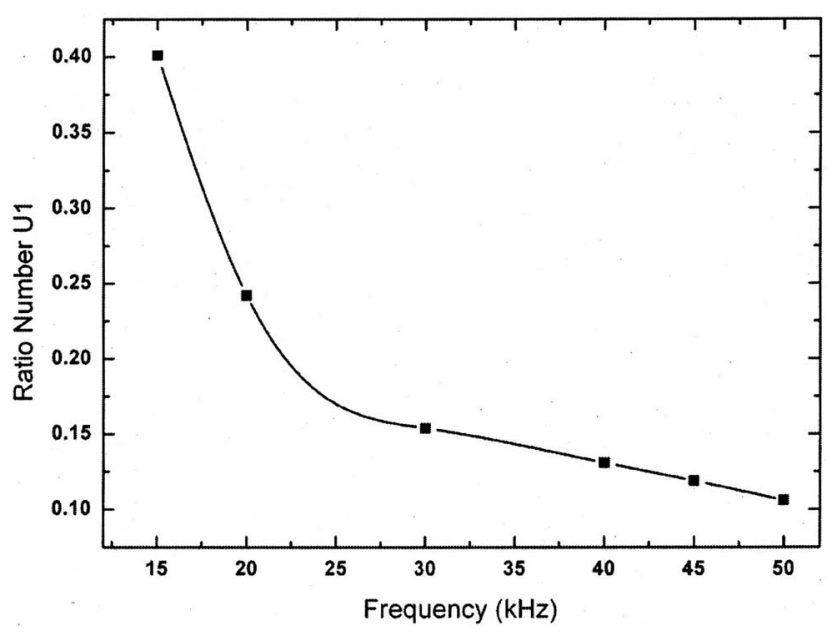

FIG. 9. The profile of ratio number $U 1$ as a function of the driving frequency with the amplitude of the applied voltage fixed at $1600 \mathrm{~V}$. 
can be used to improve the uniformity and hence the application processes of APGD.

\section{CONCLUSION}

In this paper a detailed numerical study of dielectric barrier discharges in atmospheric helium has been presented using a 2D fluid model in the cylindrical coordinate system and for cases where the gas voltage exceeds the breakdown slightly and there is only one current peak in every half cycle of the applied voltage. Through analysis of the radial distributions of electric field, charged particles and discharge current density, it has been concluded that the gas breakdown occurs firstly in the central region around the electrode axis and then again in the boundary region near the electrode edge. This spatial and temporal separation of the two breakdown events have been shown to be related to a difference in the combination of the applied voltage and the memory voltage that make up the gas voltage. Dynamic behavior of APGD in the central region are largely uniform whereas its radial profile is most significant in the boundary region. It is therefore possible to treat the central region onedimensionally and then match spatially to the boundary region. This may be used to improve computational efficiency. It has also been shown that an increase in the applied voltage or/and in the excitation frequency can enhance the radial uniformity of APGD. This offers a route to optimize APGD for their applications particularly when a large-area treatment is important.

\section{ACKNOWLEDGMENTS}

This work was supported by the National Natural Science Foundations of China under Grant No. 10275010 and Grant No. 50440430684.

${ }^{1}$ S. Kanazawa, M. Kogoma, T. Moriwaki, and S. Okazaki, J. Phys. D 21, 838 (1988).

${ }^{2}$ F. Massines et al., J. Appl. Phys. 83, 2950 (1998).

${ }^{3}$ Yu. B. Golubovskii, V. A. Maiorov, J. Behnke, and J. F. Behnke, J. Phys. D 36, 39 (2003).

${ }^{4}$ Yu. S. Akishev et al., Plasma Phys. Rep. 27, 164 (2001).

${ }^{5}$ Yu. B. Golubovskii, V. A. Maiorov, J. Behnke, and J. F. Behnke, J. Phys. D 36, 975 (2003).

${ }^{6}$ M. G. Kong and X. T. Deng, IEEE Trans. Plasma Sci. 31, 7 (2003).

${ }^{7}$ L. Mangolini et al., Appl. Phys. Lett. 80, 1723 (2002).

${ }^{8}$ F. Leipold, R. H. Stark, A. El-Habacki, and K. H. Schoenbach, J. Phys. D 33, 2268 (2000)

${ }^{9}$ X. Yuan and L. L. Raja, IEEE Trans. Plasma Sci. 31, 495 (2003).

${ }^{10}$ J. J. Shi and M. G. Kong, J. Appl. Phys. 97, 023306 (2005).

${ }^{11}$ A. A. Kulikovsky, J. Comput. Phys. 119, 149 (1995).

${ }^{12}$ A. A. Kulikovsky, J. Phys. D 27, 2556 (1994).

${ }^{13}$ A. A. Kulikovsky, J. Phys. D 27, 2564 (1994).

${ }^{14}$ A. Oda, Y. Sakai, H. Akash, and H. Sugawara, J. Phys. D 32, 2726 (1999).

${ }^{15}$ Sh. Amiranashvili and M. Y. Yu, Phys. Rev. E 66, 046403 (2002).

${ }^{16}$ X. J. Xu and D. C. Chu, Discharge Physics of Gas (Shanghai: Fudan University Press, China, 1996), p. 277 (in Chinese).

${ }^{17}$ R. Deloche, P. Monchicourt, M. Cheret, and F. Lambert, Phys. Rev. A 13, 1140 (1976).

${ }^{18}$ A. L. Ward, J. Appl. Phys. 33, 2789 (1962).

${ }^{19}$ J. W. Shon and M. J. Kushner, J. Appl. Phys. 75, 1883 (1994).

${ }^{20}$ J. Stevefelt, J. M. Pouvesle, and A. Bounchoule, J. Chem. Phys. 76, 4006 (1982).

${ }^{21}$ J. M. Pouvesle, A. Bounchoule, and J. Stevefelt, J. Chem. Phys. 77, 817 (1982).

${ }^{22}$ X. T. Deng and M. G. Kong, IEEE Trans. Plasma Sci. 32, 1709 (2004). 